\title{
Amount-dependent isotopic fractionation during compound-specific isotope analysis
}

\author{
Jochen Schmitt*,†, Bruno Glaser and Wolfgang Zech \\ Institute of Soil Science and Soil Geography, University of Bayreuth, D-95440 Bayreuth, Germany
}

Received 18 September 2002; Revised 2 March 2003; Accepted 3 March 2003

\begin{abstract}
The performance of a gas chromatography-combustion-isotope ratio mass spectrometry system (GC-C-IRMS) with respect to the dependence of $\delta^{13} \mathrm{C}$ values on the amount of sample is presented. Particular attention is paid to the localization of the amount-dependent isotopic fractionation within the system. Injection experiments with varying amounts of gases $\left(\mathrm{CO}_{2}, \mathrm{n}\right.$-hexane, and toluene) revealed that neither the detector unit nor the combustion reactor, but rather the conditions in the split/splitless injector, contributed to this effect. Although optimization of injector parameters was performed and a reduction of this adverse effect from 3 to $1 \%$ was achieved, it was not possible to eliminate isotopic fractionation completely. Consequently, additional injector parameters have to be considered and adjusted to achieve injection conditions free of fractionation. For routine analysis of the compound-specific $\delta^{13} \mathrm{C}$ analysis of different biomarkers in many environmental samples, perfect optimization may not always be reached. Therefore, in order to prevent systematic errors in the measured $\delta^{13} \mathrm{C}$ values due to different sample concentrations, it is suggested that correction for the remaining unknown amount-dependent fractionation can be made by means of co-analyzing standards of varying analyte concentrations and known $\delta^{13} \mathrm{C}$ values. Residual overall amountdependent isotope-fractionation can thus be corrected mathematically. Copyright (C) 2003 John Wiley \& Sons, Ltd.
\end{abstract}

Compound specific isotopic analysis (CSIA) of carbon at natural abundance levels $\left(\delta^{13} \mathrm{C}\right.$ measurements) is a valuable tool with applications spanning from organic geochemistry to food adulterations. ${ }^{1,2}$ One of the most utilized phenomena is the isotopic $\delta^{13} \mathrm{C}$ difference between $\mathrm{C}_{3}$ and $\mathrm{C}_{4}$ plants, about $15 \%$. To elucidate the even smaller differences of most samples, the accuracy and precision of the gas chromatography-combustion-isotope ratio mass spectrometry (GCC-IRMS) system are of great importance. In order to achieve the highest analytical quality, all contributing instrument components have to be optimized. ${ }^{3}$ In contrast to conventional dual inlet systems and IRMS systems connected to an elemental analyzer (EA), direct calibration and testing of a GC-C-IRMS system with a primary standard is not possible. ${ }^{4}$ Because of its nature as a multihyphenated technique, GC-CIRMS is inherently prone to unintended isotopic fractionation in any part of the system and at any step of the analytical procedure. In the sequence of analysis these potential isotopic fractionation factors can occur during (i) sample preparation and derivatization, (ii) injection, (iii) chromatography, (iv) combustion, (v) open split passage, (vi) ionization in the IRMS, (vii) the procedure of calibration with external and

*Correspondence to: J. Schmitt, Alfred Wegener Institute for Polar and Marine Research, Columbusstrasse, D-27568 Bremerhaven, Germany.

E-mail: jschmitt@awi-bremerhaven.de

"Current address: Alfred Wegener Institute for Polar and Marine Research, Columbusstrasse, D-27568 Bremerhaven, Germany. internal standards, and (viii) peak integration and calculation of the $\delta^{13} \mathrm{C}$ value. ${ }^{2-8}$

While fractionation effects during derivatization have been investigated at least theoretically, ${ }^{9}$ the dependence of the isotopic signal on variations in amounts of substance when using a GC-C-IRMS has attracted less attention. As pointed out by Hall et al. ${ }^{10}$ and by Glaser and Amelung ${ }^{5}, \delta^{13} \mathrm{C}$-values of individual compounds such as alkanes and amino acid enantiomers may depend on the sample amount injected. Meier-Augenstein et al. ${ }^{11}$ found that even minute chromatographic changes, such as temperature gradients, alter the isotopic compositions derived for the peaks. Generally, the term non-linearity is used to describe this effect. ${ }^{12,13}$ In this paper we follow the suggestion of Hall et al. ${ }^{10}$ and use amount dependence, since 'linearity' usually describes a 1st order polynomial function. Up to now, this undesired drift with signal size ${ }^{4}$ has been corrected using logarithmic functions that reflect changes in $\delta^{13} \mathrm{C}$ upon the injection of different amounts of standards. ${ }^{5}$ Clearly, the final goal of any CSIA method should be to find the right parameter and technique combination to eliminate amount-dependent isotopic fractionation. Since analyte concentrations in environmental samples vary considerably between different samples, amount-dependence poses a threat to accuracy. If this systematic bias in a set of samples is not detected, data interpretation may give misleading results.

One general strategy to avoid this problem is to measure the concentration of the analyte in advance and then adjust the concentration by dilution or adapting the amount of 
sample processed. Though this has to be done with any sample and analyte to match the exact concentration of the used standard, this procedure is only feasible when focusing on just one analyte. This procedure is analogous to sample size adjustment with a correction algorithm often recommended and necessary for EA-IRMS analyses. ${ }^{13,14}$ For GC-CIRMS analysis, where one is interested in several analyte compounds each at its own concentration, adjustment of sample amounts and subsequent measurement of the compounds at individual dilutions separately is tedious and costly and often limited by the amount of available sample material. Therefore, we suggest to determine the amount-dependence of the $\delta^{13} \mathrm{C}$ values of target compounds over the whole dynamic range using standards, and to find best-fit functions for the relationship between $\delta^{13} \mathrm{C}$ and the sample amount injected. Such a procedure has already been suggested by Hall et al., ${ }^{10}$ who investigated the amountdependence at extremely low analyte amounts.

However, the phenomenon of signal dependent $\delta^{13} \mathrm{C}$ is not restricted to only low sample analyte amounts, but seems to occur throughout the whole dynamic range. ${ }^{5}$ Therefore, the objective of this study was (i) to detect possible sources of unintended fractionation within the GC-C-IRMS system and (ii) to optimize subsequently the measurement parameters in order to improve the accuracy of GC-C-IRMS measurements of samples with a wide variation of analyte concentrations. We have to point out that it is not the aim of this study to provide a comprehensive scheme for a perfect optimization of the GC-C-IRMS system. Books and some papers dealing with this are already available. ${ }^{2,3,11,15}$ The present aim is to emphasize a problem which we feel gets too little attention from the CSIA community, although it poses a great risk of hidden systematic errors deteriorating the accuracy of compound-specific stable isotope measurements.

The localization of the causes of amount-dependence is also crucial for decisions as to which calibration method is applicable and most accurate. To achieve this, a general procedure to systematically check for amount-dependence caused by each part of the entire GC-C-IRMS system is presented, along with optimized parameters and possible mathematical corrections. Clearly, this end-of-pipe correction is not a substitute for a proper optimization of all the instrument parameters, but should serve to help prevent systematic errors with different sample concentrations in cases when isotopic fractionation is not totally avoidable.

Although this study focused on lignin-derived phenols, the effect described was also observed for other compounds such as amino acids, amino sugars, and phospholipid fatty acids. However, these results are not shown here.

\section{EXPERIMENTAL}

\section{Instruments}

All experiments were performed using a GC-C-IRMS system consisting of a Trace GC 2000 gas chromatograph (Thermo Finnigan MAT, Bremen, Germany) equipped with a Programmable Temperature Vaporization (PTV) injector. The injector was used with a gooseneck liner for splitless injection (total length $10.5 \mathrm{~cm}, 5 \mathrm{~mm}$ i.d. for $9.2 \mathrm{~cm}$ ), which was deactivated with $5 \%$ dimethylchlorosilane in toluene for at least one day; no glass wool packing was used. Chromatographic separation used an HP-5 MS column $(30 \mathrm{~m} \times 0.25 \mathrm{~mm} \times$ $0.25 \mu \mathrm{m}$ film thickness of a cross-linked copolymer of $5 \%$ diphenyl- and 95\% dimethyl-polysiloxane; Agilent Technologies, Palo Alto, CA). The connection to the Combustion Interface III (Thermo Finnigan MAT, Bremen, Germany) was accomplished with a borosilicate press fit Y-splitter (IVA, Meerbusch, Germany). Isotope ratios were measured using a Delta ${ }^{\text {plus }}$ IRMS (Thermo Finnigan MAT, Bremen, Germany). The tune settings of the instrument were optimized for isotopic linearity. Details concerning the calibration using $\mathrm{CO}_{2}$ as a secondary standard are published elsewhere. ${ }^{5}$ The temperature of the combustion unit was set to $940^{\circ} \mathrm{C}$ during all experiments. Helium 4.6 (Riessner, Lichtenfels, Germany) was used as carrier gas at a constant carrier gas flow rate of $0.8 \mathrm{~mL} \mathrm{~min}^{-1}$. The injector temperature was always set at $250^{\circ} \mathrm{C}$.

For $\mathrm{CO}_{2}$ and n-hexane/toluene experiments, injection was accomplished manually using a gas-tight syringe (Exmire Micro Syringe MS GF10, ITO Corporation, Japan). Injection of the phenol standards was done using an autosampler (AS 2000, Thermo Finnigan MAT, Bremen, Germany) with two different types of $10-\mu \mathrm{L}$ syringes, one with $50 \mathrm{~mm}$ and a second with $70 \mathrm{~mm}$ needle length (both from Hamilton, Bonaduz, Switzerland).

\section{Experiments for localization of amount dependence}

\section{Preparation and injection of phenol standards}

The overall amount-dependence was tested by injecting varying amounts of derivatized phenol standards, similar to the procedure suggested by Glaser and Amelung ${ }^{5}$ for amino acid enantiomers. A stock solution $\left(1000 \mathrm{mg} \mathrm{L}^{-1}\right)$ of the following phenols was prepared in methanol p.a.: ethylvanillin, vanillin, vanillic acid, syringealdehyde, syringic acid, p-coumaric acid, and ferulic acid. From this stock solution an aliquot of $100 \mu \mathrm{L}$ was evaporated in a vial under a gentle stream of nitrogen to remove the solvent. Derivatization was performed by adding $400 \mu \mathrm{L}$ pyridine and $800 \mu \mathrm{L} \mathrm{N}, \mathrm{O}-$ bis-(trimethylsilyl)-trifluoroacetamide (BSTFA). After gentle shaking, reaction was allowed to proceed at ambient temperature for one hour. From the derivatized solution, aliquots of $250,100,100,50,25$ and $10 \mu \mathrm{L}$ were taken and diluted with $0,100,200,200,200$, and $200 \mu \mathrm{L}$ of a mixture of pyridine:BSTFA (1:2), respectively. The corresponding concentrations were $83,42,28,17,9$ and $4 \mathrm{ng} \mu \mathrm{L}^{-1}$. The solution was stable for up to 3 days. Preparing one derivatized stock solution that is diluted subsequently was preferred over the derivatization of separate vials with varying amounts of standard stock solution because the latter method changes the stoichiometry of analyte to derivatizing reagent, thus poses the potential risk of kinetic fractionation.

This series of standards with increasing concentrations was measured in triplicate. This cyclic routine was preferred over measuring each concentration three times immediately one after the other, thus eliminating errors caused by a temporal drift during the long measurement time of a standard sequence. Although analytical precision in terms of standard deviation may be reduced in this way, the 
dependence of $\delta^{13} \mathrm{C}$ values on injection amount can be detected more reliably. A constant sample volume of $1 \mu \mathrm{L}$ was injected using the syringe with $50 \mathrm{~mm}$ needle length. Amounts ranging from 4 to 83 ng were injected using splitless mode, with a splitless time of $1 \mathrm{~min}$. For the autosampler injections we used the sample in the needle technique with $0 \mathrm{~s}$ pre-injection time and $1 \mathrm{~s}$ post injection delay, and with injection speed at maximum. A ramped temperature program was used, with $0.5 \mathrm{~min}$ isothermal at $100^{\circ} \mathrm{C} ; 100^{\circ} \mathrm{C}$ to $250^{\circ} \mathrm{C}$ at $5^{\circ} \mathrm{C} \mathrm{min}^{-1} ; 250^{\circ} \mathrm{C}$ to $300^{\circ} \mathrm{C}$ at $30^{\circ} \mathrm{C} \mathrm{min}^{-1} ; 5 \mathrm{~min}$ isothermal at $300^{\circ} \mathrm{C}$.

\section{$\mathrm{CO}_{2}$ injection via the reference gas open split (standard on/off)}

The most direct introduction of $\mathrm{CO}_{2}$ into the ion source is via the reference gas open split. This can be done repeatedly via the ISODAT software (Thermo Finnigan MAT, Bremen, Germany) and is known among Thermo Finnigan IRMS users as 'standard on/off'. Automatically controlled by valves, $\mathrm{CO}_{2}$ pulses of $20 \mathrm{~s}$ length are introduced into the reference gas open split and transferred to the ion source of the IRMS. Between each pulse a pause of $30 \mathrm{~s}$ was allowed, and 18 on/off-cycles were measured within a sequence. Two different modes of this standard on/off procedure were applied. First, $\mathrm{CO}_{2}$ pressure was kept constant at $150 \mathrm{kPa}$. In the second experiment, the $\mathrm{CO}_{2}$ flow was manually altered via the pressure regulator under the control of a manometer. To prevent $\mathrm{CO}_{2}$ pressure fluctuation during the measurement, the pressure was changed immediately after each on/off-cycle, thus allowing about $25 \mathrm{~s}$ for equilibration of the flow conditions. The procedure was started at $250 \mathrm{kPa}$ with calibration and four pulses for stability test; afterwards the pressure was reduced to $200,150,100,50$, and $25 \mathrm{kPa}$, followed by stepwise increases to 50,100 and $250 \mathrm{kPa}$.

\section{$\mathrm{CO}_{2}$ injection via the split/splitless injector}

$\mathrm{CO}_{2}$ from a high-pressure cylinder, used as the working standard, flowed several minutes via a drainage tube through a septum-capped 1-ml vial to displace all air. From this vial volumes from 0.2 to $10 \mu \mathrm{L}$ were sampled with a gas tight syringe and injected repeatedly into the injector of the GC. Injections were performed at split ratios of 12:1, 60:1, and 120:1. During this experiment the temperature of the GC was held isothermal at $100^{\circ} \mathrm{C}$.

\section{Injection of n-hexane and toluene gas mixture}

To prepare a gas mixture of n-hexane or toluene in helium, a 1-ml vial was flushed and filled with $\mathrm{He} 4$.6. Into this vial $5 \mu \mathrm{L}$ of $n$-hexane or toluene were injected. After equilibrating for one hour at ambient temperature, the solvent completely evaporated giving a homogenous gas mixture. Analogous to the $\mathrm{CO}_{2}$ experiment, volumes of 1 to $10 \mu \mathrm{L}$ were injected into the GC in triplicate at two different split ratios (12:1 and 120:1). The GC temperature was held at $200^{\circ} \mathrm{C}$ to minimize interaction with the stationary phase and thus avoiding any chromatographic isotope effect. In fact we were unable to detect any measurable retention time delay between $\mathrm{CO}_{2}$ and hexane or toluene. Additionally, our assumption that hexane and toluene do not noticeably interact with the stationary phase under these conditions was corroborated by the intensity ratio for the signals at $m / z 45 / 44$. The two compounds did not show the classic S-shaped 'up-and-down' shape of the GC peaks for the $m / z 45 / 44$ intensity ratio, which is normally attributed to chromatographic separation of isotopomers of a compound interacting with the stationary phase.

\section{Optimizing injector parameters with phenol solution}

In order to optimize injector parameters, derivatized phenol standards were injected as described above. To check the influence of the syringe type, the autosampler was alternately equipped with a syringe with $50 \mathrm{~mm}$ or $70 \mathrm{~mm}$ needle length. With each syringe, the set of derivatized standards was measured in duplicate with the temperature ramped GC conditions described above.

In the second experiment the phenol standards were injected at three different splitless times, namely, $0.5 \mathrm{~min}$, $1 \mathrm{~min}$, and $3 \mathrm{~min}$. Injection was done using the 70-mm needle, but all other parameters were the same as in the previous experiment.

\section{RESULTS AND DISCUSSION}

As already indicated by Glaser and Amelung, ${ }^{5} \delta^{13} \mathrm{C}$ values of amino acid enantiomers may depend on the injection amount. In order to check whether lignin-derived phenols exhibit the same behavior, we measured $\delta^{13} \mathrm{C}$ values of derivatized standard solutions with increasing concentrations of the seven phenol standards. Figure 1 illustrates $\delta^{13} \mathrm{C}$ values measured with the GC-C-IRMS system set to standard conditions. Three characteristics are discernible: (i) within the range of 9 to $80 \mathrm{ng}$ more than $2 \%$ deviation can be observed; (ii) individual compounds show individual extents of fractionation; (iii) these extents of fractionation are related in a highly non-linear manner to the sample amount injected (Fig. 1). In order to find the cause(s) of the amount-dependence within the multi-component GC-C-IRMS system, it is useful to conduct experiments with the least number of

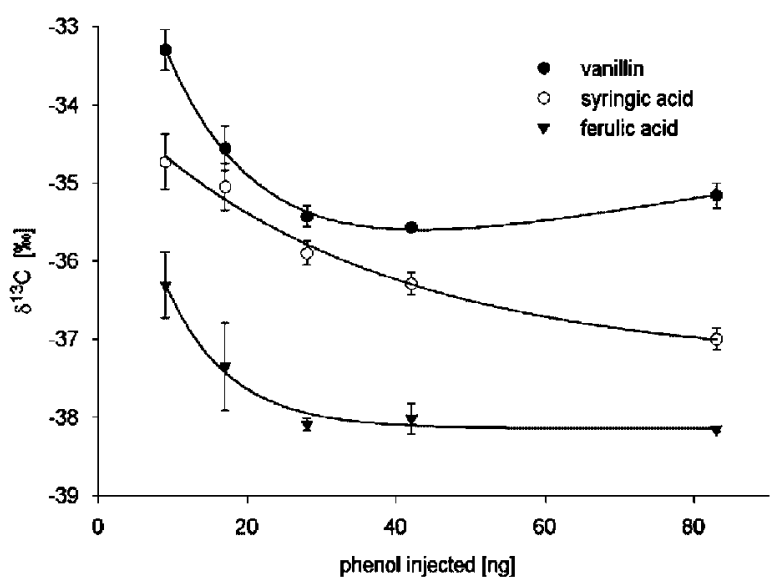

Figure 1. $\delta^{13} \mathrm{C}$ amount-dependence of three phenols. Injection amounts range from 9 to $83 \mathrm{ng}$ of underivatized phenol which correspond to 0.6 to $7 \mathrm{nmol} \mathrm{C}$ of the derivatized phenol. IRMS signal response for $m / z 44$ was between 0.2 to $3 \mathrm{~V}$, thus within the range considered to yield signal-independent $\delta^{13} \mathrm{C}$ values. Error bars represent the standard error of the mean $(n=3)$. 
unknown variables. Therefore, we conducted the tests backwards, starting with the IRMS followed by the combustion unit and ending with the injector.

\section{IRMS-injector: $\mathrm{CO}_{2}$ experiments}

The first experiment aimed at determining a possible amount-dependence of the IRMS detector by injection of $\mathrm{CO}_{2}$ directly into the carrier gas stream entering the IRMS. Actually, there are several ways to introduce $\mathrm{CO}_{2}$ into the ion source. The most common and easiest way is via the reference gas open split reserved for the standard on/off procedure. Standard on/off experiments are routinely performed daily to test for signal stability over time. The signal stability of the utilized system was satisfactory, showing no systematic temporal variation of the measured ${ }^{13} \mathrm{C} /{ }^{12} \mathrm{C}$ ratios, and standard deviations of ten replicate measurements were usually $<0.02 \%$. By stepwise changing the $\mathrm{CO}_{2}$ pressure it is possible to change the dilution ratio of $\mathrm{CO}_{2}$ in $\mathrm{He}$, thus providing the IRMS with different $\mathrm{CO}_{2}$ amounts. The results of this $\mathrm{CO}_{2}$ standard on/off experiment are shown in Fig. 2. Varying the $\mathrm{CO}_{2}$ pressure from 20 to $250 \mathrm{kPa}$, corresponding to signal amplitudes for $m / z 44$ amplitude of 0.3 to $5.5 \mathrm{~V}$, causes a $\delta^{13} \mathrm{C}$ shift of $>1 \%$.

At first sight this procedure seemed to be an ideal way to circumvent the GC-C system and thus to check the IRMS directly. However, changing the pressure by manipulating the pressure regulator may alter the flow conditions within the pressure regulator and the capillary which supplies the reference gas open split. This can lead to isotopic fractionation resulting in a dependence of the $\delta^{13} \mathrm{C}$ signal on the $\mathrm{CO}_{2}$ amount. ${ }^{16}$ Hence, the interpretation of the observed amount-dependence as an effect of the IRMS detector is ambiguous. Additionally, in our case it could be unambiguously shown that the IRMS detector is properly adjusted to 'linearity' by connecting the IRMS to an elemental analyzer and by repeating the standard on/off experiment; thus we had another indication that our source tune settings were properly adjusted.

To introduce $\mathrm{CO}_{2}$ or other gases at any part of the GC-CIRMS system, a reference gas inlet module that permits both internal isotopic calibration and control of the performance of the combustion reactor, has been proposed. ${ }^{17}$ Other possibilities to introduce $\mathrm{CO}_{2}$ into the IRMS are injection of $\mathrm{CO}_{2}$ via the split/splitless injector at the GC, and the injection of organic substances with subsequent combustion to $\mathrm{CO}_{2}$ in the reactor. With the latter technique all possible fractionation processes during injection, incomplete combustion, and chromatography, could occur as discussed above. Therefore, to identify isotopic fractionation linked to the injection of $\mathrm{CO}_{2}$, in the next step $\mathrm{CO}_{2}$ was injected via the split/splitless injector: The advantage of this procedure relative to the above-mentioned standard on/off method is that the $\mathrm{CO}_{2}$ passes through the GC column and the combustion reactor and finally reaches the IRMS detector under almost exactly the same flow conditions as the analytes do. Because $\mathrm{CO}_{2}$ is not subjected to chromatographic separation in the HP-5 column used, isotopic fractionation within the $\mathrm{CO}_{2}$ peak is assumed to be negligible. Therefore, introducing $\mathrm{CO}_{2}$ to the ion source in this way is comparable to the standard on/off procedure but prevents fractionation within the pressure regulator due to changing flow conditions and pressure pulses.

To get integratable, sharp $\mathrm{CO}_{2}$ peaks, injection had to be done in split-mode. Figure 3 shows the results of the $\mathrm{CO}_{2}$ injection experiments with varying $\mathrm{CO}_{2}$ amounts. It can be seen that there was no amount-dependence at high split ratios. Therefore, the varying amounts of $\mathrm{CO}_{2}$ reaching the ion source are measured correctly at a split ratio of 120:1. Therefore, ionization and detection within the IRMS were not responsible for the observed amount-dependence of $\delta^{13} \mathrm{C}$ values observed for the phenols. The second result of this experiment was that low split ratios either lead to isotopic fractionation within the injector system, or that the observed variation reflects the effect of modified peak parameters, e.g. height/area ratio, peak broadening, start and end slopes. ${ }^{15}$ The $\mathrm{CO}_{2}$ tests proved that, at least at a certain injector setting, it was possible to measure amount-independent ${ }^{13} \mathrm{C} /{ }^{12} \mathrm{C}$ ratios. Thus, using this setting, further checks were performed on the other components of the GC-C-IRMS system, the combustion unit and the injector.

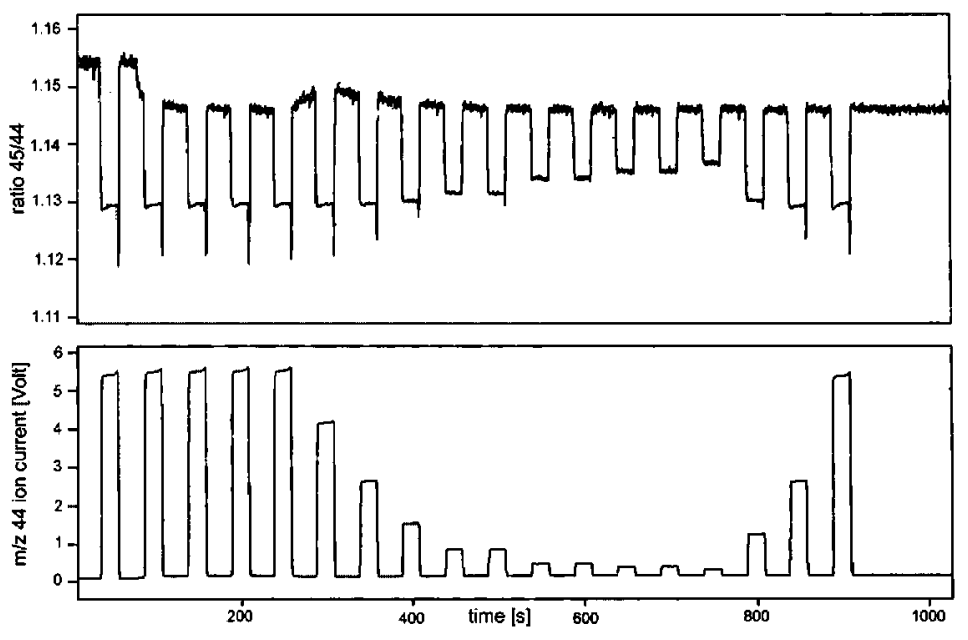

Figure 2. Standard on/off experiment with the $\mathrm{CO}_{2}$ pressure varying from 20 to $250 \mathrm{kPa}$. An isotopic fractionation of $>1 \%$ is observed for a variation of IRMS response at $m / z 44$ between 0.3 and $5.5 \mathrm{~V}$. 

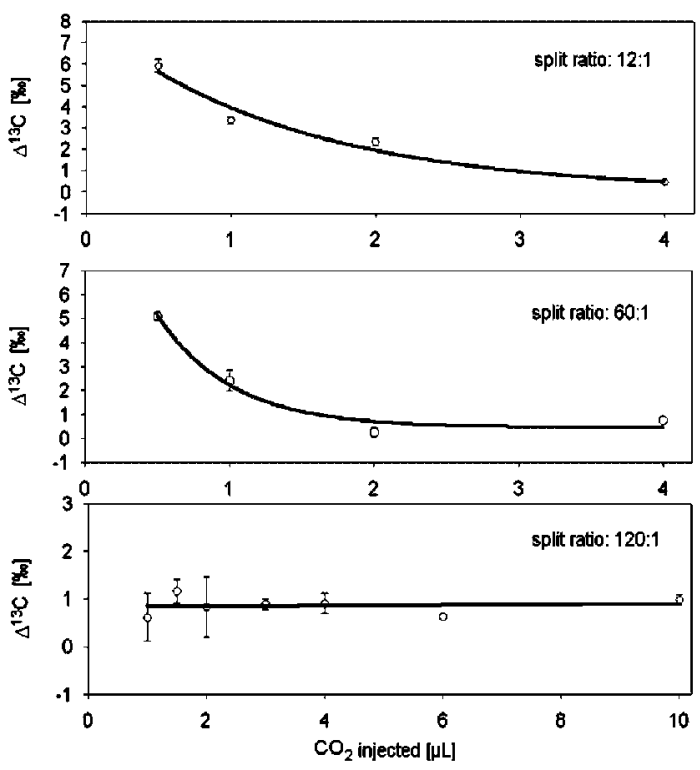

Figure 3. Injection experiment of variable $\mathrm{CO}_{2}$ volumes at three different split ratios. The amount-dependence of the $\delta^{13} \mathrm{C}$ value is influenced by the flow rate within the liner. At the highest flow rate the isotopic discrimination is minimal and thus the $\delta^{13} \mathrm{C}$ values are amount-independent. Measured $\delta^{13} \mathrm{C}$ values are given as the difference relative to the calibration via the reference gas open split $(\Delta)$. Error bars indicate the standard error of the mean $(n=3)$.

\section{Checking the combustion unit: n-hexane and toluene experiments}

Since the introduction of online combustion interfaces with $\mathrm{CuO} / \mathrm{NiO}$ wires, it has been known that aromatic compounds are less easily combustible than alkanes. ${ }^{6}$ Therefore, we suspected that the amount dependence of the measured phenol compounds might be due to incomplete combustion. In order to test the performance of the combustion reactor, varying amounts of toluene and n-hexane as model substances for aromatic and aliphatic compounds, respectively, were injected as head space samples at the same high split ratio as in the previous $\mathrm{CO}_{2}$ experiments. Provided that combustion is incomplete, the $\delta^{13} \mathrm{C}$ values of larger sample size should deviate from those measured for smaller sample amounts. In addition, toluene and n-hexane should also behave differently due to different combustion properties. Figure 4 shows clearly that combustion was not responsible for the amount-dependence, since the increasing sample amounts injected had no influence on the $\delta^{13} \mathrm{C}$ values and the two model compounds behaved similarly. From these experiments it can be concluded that neither the IRMS detector nor the combustion unit caused the amount-dependence of the $\delta^{13} \mathrm{C}$ values for the phenols.

Comparison of the results of the $\mathrm{CO}_{2}$ and n-hexane/ toluene experiments at the split ratio of 12:1 shows that the magnitude of isotopic fractionation within the injector is characteristic of each substance. Whereas $\mathrm{CO}_{2}$ undergoes a fractionation of up to $6 \%$, the deviation of n-hexane was only about $1 \%$, and varying amounts of toluene yielded constant $\delta^{13} \mathrm{C}$ values. Thus, even optimizing a GC-C-IRMS system for one single analyte does not necessarily mean that this is the case for other analytes. The small but measurable $\delta^{13} \mathrm{C}$

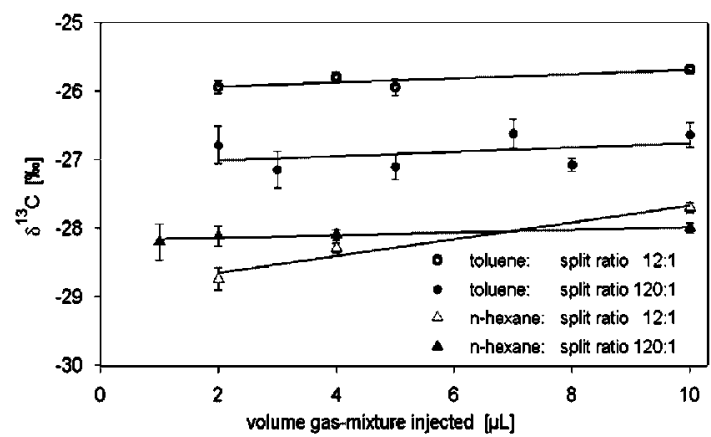

Figure 4. Measured $\delta^{13} \mathrm{C}$ values from repeated injections of increasing volumes of toluene and $n$-hexane vapor at two different split ratios. At a split ratio of $120: 1$, the $\delta^{13} \mathrm{C}$ of both compounds showed no amount-dependence, indicating no fractionation within the oxidation reactor, whereas $n$-hexane showed moderate amount-dependent isotopic fractionation at a split ratio of 12:1. Response of the IRMS at $m / z 44$ was 1 to $9 \mathrm{~V}$ for the split ratio $12: 1$, and 0.2 to $8 \mathrm{~V}$ for the $120: 1$ split ratio. Error bars indicate the standard error of the mean $(n=3)$.

difference for toluene at the two split ratios probably points to a thermodynamic fractionation effect as it is independent of the sample amount. Thus, our further efforts concentrated on injector conditions for the analysis of lignin phenols.

\section{Optimizing injector conditions}

As already pointed out by Meier-Augenstein ${ }^{11}$ and also deduced from the above experiments, flow conditions in the injector influence the isotopic fractionation during split injection. Elimination of the amount-dependence in both the $\mathrm{CO}_{2}$ and the n-hexane/toluene experiments was only possible at high split ratios which are not applicable for measuring environmental samples. Concentrations, especially of lignin phenols, are often low and therefore have to be analyzed in splitless mode. Furthermore, both the $\mathrm{CO}_{2}$ and the n-hexane/toluene experiments essentially excluded isotopic fractionation during chromatographic separation since the GC temperature was at 100 and $200^{\circ} \mathrm{C}$, respectively. Additionally, the injection of a liquid solution which has yet to be vaporized within the liner compared to a sample already in the gaseous state clearly poses quite different fractionation conditions. ${ }^{15}$ Therefore, optimization of the injection parameters was subsequently performed under realistic analytical conditions for phenol separation, i.e. splitless mode, temperature programmed GC conditions, and a constant flow rate of $0.8 \mathrm{~mL} \mathrm{~min}{ }^{-1}$. The use of splitless injection reduced the amount-dependence for amino acid enantiomers, ${ }^{5}$ but an increasing number of publications report use of on-column injection to prevent any amount-dependence. According to Meier-Augenstein ${ }^{3}$ and Grob, ${ }^{15}$ splitless injection with opening of the splitter after $10 \mathrm{~s}$ is recommended.

Since it was not the aim of this study to provide a scheme for overall optimization of each parameter of the injection process, only the following parameters could be checked for influence on amount-dependence for the phenols: needle length and duration of the splitless time. Referring to the extensive work of $\mathrm{Grob}^{15}$ on processes during injection, incomplete vaporization of the solutes within the needle and fractionation of the solutes might also contribute to the 
observed isotopic fractionation (amount-dependence). Therefore, optimization of the injection technique (e.g. cold needle, hot needle, air plug or the sandwich method) could offer further potential to eliminate the amount-dependence.

\section{Injector parameters}

Two different needle lengths were tested with different concentrations of derivatized phenols. Both versions led to amount-dependence as exemplified for vanillin (Fig. 5). Interestingly, the curves characteristic of the relationship between the sample amount injected and fractionation were different which indicates that minor changes in the injection system influence the isotopic fractionation, a fact already noted by Meier-Augenstein ${ }^{11}$ and Grob. ${ }^{15}$ Further, use of the syringe with the $70-\mathrm{mm}$ needle reduced the amountdependence of $\delta^{13} \mathrm{C}$ of vanillin from 2 to $1 \%$. The longer needle injects the sample closer to the GC column inlet than the shorter needle, thus the vapor cloud is more restricted to the end of the liner and therefore transmission of the solutes is faster and more quantitative. These results are consistent with the observations of Grob. ${ }^{15}$ Furthermore, it can be deduced that the volume of the liner combined with the carrier gas flow rate of $0.8 \mathrm{~mL} \mathrm{~min}^{-1}$ is not adjusted appropriately. We suspect that either a reduction of the liner volume or an increase of the flow rate, or a combination of both, would enhance quantitative vapor transfer from the liner to the column. However, for practical uses such as the separation of individual lignin phenols, it turned out that this carrier gas flow yields the best separation. Additionally, for very low phenol concentrations, injection volumes larger than $1 \mu \mathrm{L}$ may be necessary. In these cases, small volume liners might not be appropriate. ${ }^{15}$

Due to the fact that isotopic fractionation is expected when substance is lost, longer splitless times probably lead to reduced amount-dependence because less sample is lost

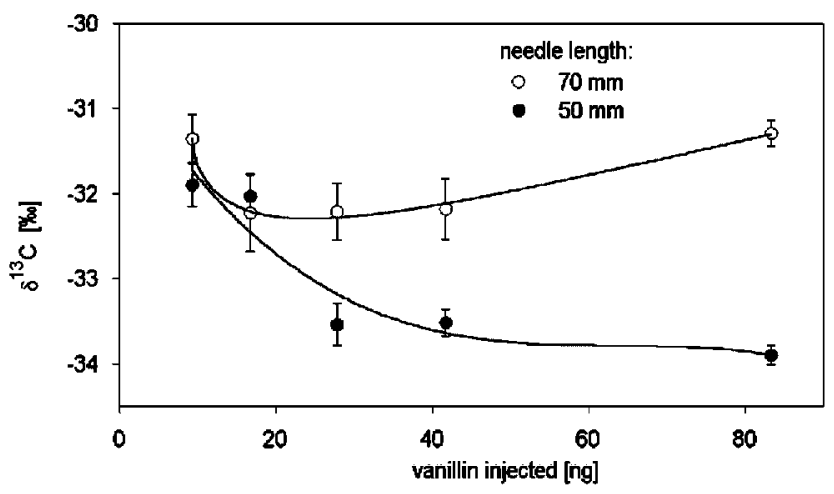

Figure 5. Influence of the needle length on the amountdependence of the $\delta^{13} \mathrm{C}$ value of derivatized vanillin during splitless injection. Both extent and characteristics of the amount-dependent fractionation are different, with the shorter needle inducing more pronounced fractionation with an amplitude of $2 \%$ in the $\delta^{13} \mathrm{C}$ value. In contrast, fractionation for the longer needle-type is less, but exhibits a more complex relationship between $\delta^{13} \mathrm{C}$ and the amount of sample injected. IRMS response for $m / z 44$ response was 0.2 to $4 \mathrm{~V}$. Error bars indicate the standard error of the mean $(n=3)$.

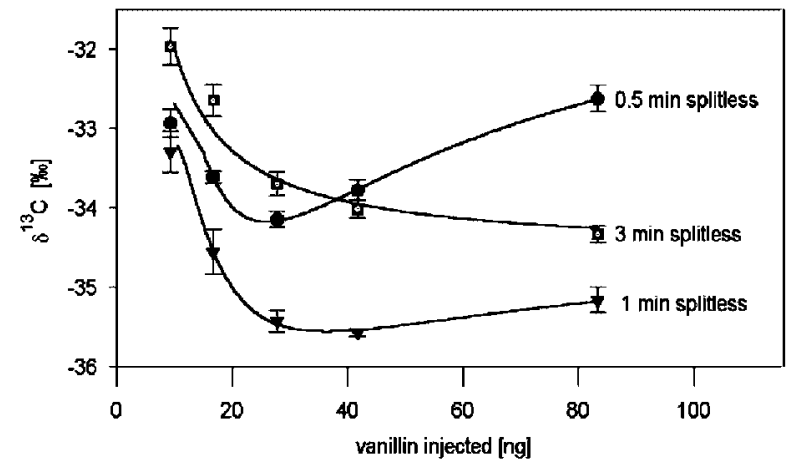

Figure 6. Influence of splitless time on amount-dependence of $\delta^{13} \mathrm{C}$ of vanillin. Longer splitless time leads to reduced amount-dependent fractionation within the injector. IRMS signal for $m / z 44$ ranged from $0.2 \mathrm{~V}$ at $9 \mathrm{ng}$ injection amount to $4 \mathrm{~V}$ at $80 \mathrm{ng}$. Error bars represent the standard error of the mean $(n=3)$.

through the splitter. However, all three splitless times applied resulted in a dependence of the $\delta^{13} \mathrm{C}$ value on the amount injected (Fig. 6). Although the maximum amountdependence was about $2 \%$ in all three variants, the functional characteristics of the relationships between the fractionation and the amount of sample injected were different. The shorter the time the splitter is closed, the more pronounced the functional complexity of the resulting dependency of the $\delta^{13} \mathrm{C}$ on the sample amount. Whereas at both 0.5 and $1 \mathrm{~min}$ splitless times at least a 3rd order polynomial curve fitting was required to properly describe the functional relationship between fractionation and sample amount, a simple logarithmic fit, similar to that suggested for amino acid enantiomers, ${ }^{5}$ was adequate for the results of the 3 min splitless time experiment. Other compounds even exhibit a linear dependency (see results of the next section). Such less complicated dependencies require fewer data points and are easier to describe. In contrast with the findings of MeierAugenstein $^{3}$ we could not detect any visible deterioration of the chromatographic performance due to the long splitless time applied. The fact that even 3 min splitless time is not sufficient to prevent isotopic fractionation points to two possible reasons. First, the carrier gas flow setting of $0.8 \mathrm{~mL}$ $\min ^{-1}$ might be too slow for the liner volume, so that backward mixing of the solute vapor and loss via the septum purge is possible. Second, the different splitless times may modify chromatographic peak separation due to band broadening with increasing splitless time, especially at longer than 3 minutes. A retention gap of $1 \mathrm{~m}$ may improve chromatographic separation and performance due to refocussing the solute band. ${ }^{3}$ The influence of chromatographic parameters, e.g. temperature gradients, on isotopic fractionation, has been outlined by Meier-Augenstein et al. ${ }^{11}$

\section{Correction for amount-dependence}

For calibration and correction of the remaining amountdependence we suggest the following procedure. To try to fulfill the requirements of identical treatment (IT), ${ }^{4}$ we calibrated the individual sample peaks of each chromatogram against the internal standard (ethylvanillin) of known isotopic composition. Since we plan to apply the method to 
soil and plant samples, complex chromatograms with possible co-elution of interfering compounds are expected. Therefore, depending on the chromatogram, the analyte peaks were either calibrated to the working standard $\mathrm{CO}_{2}$ or to the internal standard. Further, the correction for amountdependence has to be established with external standards. Either a logarithmic or a linear regression was fitted to at least 10 standards of different concentrations. The amount-dependent fractionation of the first eluting aldehyde compounds followed logarithmic curves while the acids with longer retention times showed a linear relationship between isotopic fractionation and the amount injected (Fig. 7).

As the measured phenols were derivatized with BSTFA, which is composed of two different types of carbon, calculation of the isotopic composition of the trimethylsilyl (TMS) group cannot be done directly via mass balance. ${ }^{9}$ The $\delta^{13} \mathrm{C}$ value of the TMS group has to be calculated by measuring the underivatized phenol with EA-IRMS and the derivative with GC-C-IRMS. ${ }^{18}$ Unfortunately, the calculation is further complicated since the $\delta^{13} \mathrm{C}$ value of the derivative is a function of the amount injected. To avoid calculating a signaldependent $\delta^{13} \mathrm{C}$ value for the TMS group, the $\delta^{13} \mathrm{C}$ value of the derivative at a IRMS signal intensity of $1 \mathrm{~V}$ for $m / z 44\left(\mathrm{~V}^{44}\right)$
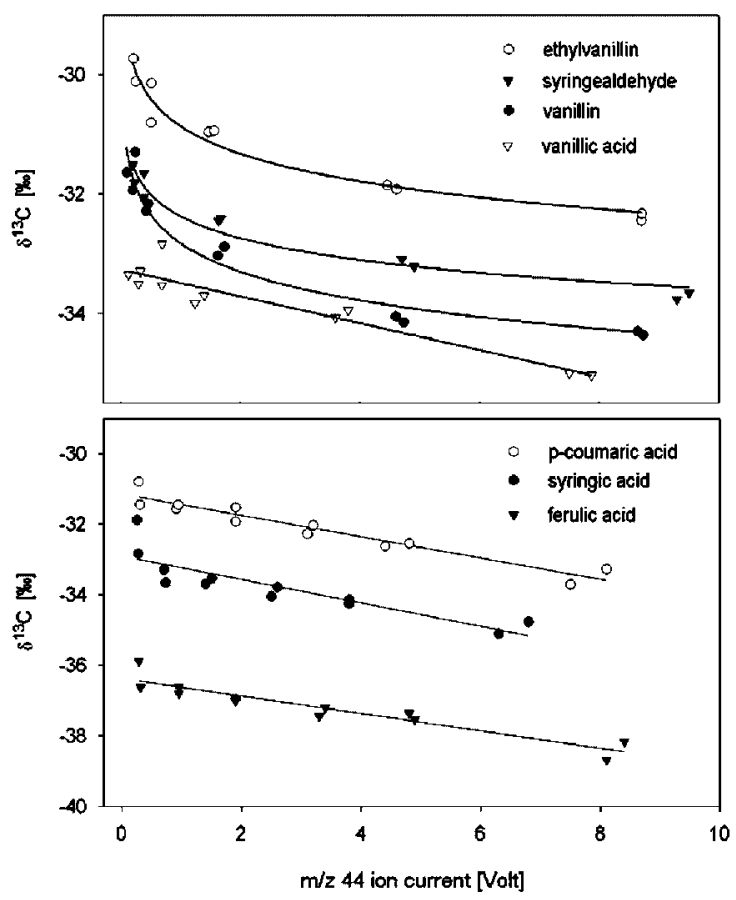

Figure 7. Regression functions between $\delta^{13} \mathrm{C}$ and the amount of sample injected for seven phenol compounds after optimization of the injection parameters. Amountdependence of the compounds is between 1 and $2 \%$, with the earlier eluting aldehydes exhibiting a logarithmic amountdependent fractionation. The acids with longer retention times usually exhibited a linear amount-dependence of $\delta^{13} \mathrm{C}$; however, the extent of the amount-dependence varies between different experimental sequences. Therefore, the amount dependence of the $\delta^{13} \mathrm{C}$ needs to be readjusted sequence by sequence, especially after changing the liner or replacing the GC column. The intensity for $\mathrm{m} / \mathrm{z} 44$ corresponds to injection amounts of 0.7 to $7 \mathrm{nmol}$ carbon.
Table 1. Equations of logarithmic and linear regression fits of the relationship between the sample amount injected ( $x$, expressed as signal intensity in $\mathrm{V}$ ) and the $\delta^{13} \mathrm{C}(\mathrm{y})$ of the seven phenol compounds shown in Fig. 7

\begin{tabular}{llc}
\hline Compound & Equation & $\mathrm{r}^{2}$ \\
\hline Vanillin & $\mathrm{y}=-0.69 \ln (\mathrm{x})-32.82$ & 0.97 \\
Ethylvanillin & $\mathrm{y}=-0.67 \ln (\mathrm{x})-30.86$ & 0.98 \\
Syringealdehyde & $\mathrm{y}=-0.53 \ln (\mathrm{x})-32.87$ & 0.98 \\
Vanillic acid & $\mathrm{y}=-0.22 \mathrm{x}-33.26$ & 0.94 \\
Syringic acid & $\mathrm{y}=-0.33 \mathrm{x}-32.90$ & 0.76 \\
p-coumaric acid & $\mathrm{y}=-0.30 \mathrm{x}-31.14$ & 0.93 \\
Ferulic acid & $\mathrm{y}=-0.24 \mathrm{x}-36.39$ & 0.88 \\
\hline
\end{tabular}

was arbitrarily selected. This value was obtained from the functions (Table 1) derived from regression fits (Fig. 7) for each compound. This is accomplished by inserting $1 \mathrm{~V}$ into the linear or logarithmic regression fits (Eqns. 1 and 2) of the variable injection amounts (see Fig. 7 with regression curves).

$$
\begin{gathered}
\delta^{13} \mathrm{C}_{\text {derivative 1V }}=\mathrm{a}_{\operatorname{lin}}(1)+\mathrm{b}_{\text {lin }} \\
\delta^{13} \mathrm{C}_{\text {derivative 1V }}=\mathrm{a}_{\log } \ln (1)+\mathrm{b}_{\log }
\end{gathered}
$$

To correct the $\delta^{13} \mathrm{C}$ values of sample derivatives for amount dependence, Eqn. 3 or 4 for logarithmic or linear fit, respectively, has to be applied by inserting the sample value of $\mathrm{V}^{44}$. Thus free from amount-dependence, the obtained $\delta^{13} \mathrm{C}_{\text {corrected }}$ value of the derivative is then inserted into the isotopic mass balance to calculate the $\delta^{13} \mathrm{C}$ value of the initial molecule (application of isotopic mass balance is described elsewhere). ${ }^{18}$

$$
\begin{gathered}
\delta^{13} \mathrm{C}_{\text {corrected }}=\delta^{13} \mathrm{C}_{\text {actural }}-\mathrm{a}_{\operatorname{lin}}\left(\mathrm{V}^{44}-1\right) \\
\delta^{13} \mathrm{C}_{\text {corrected }}=\delta^{13} \mathrm{C}_{\text {actural }}-\mathrm{a}_{\log } \ln \left(\mathrm{V}^{44}\right)
\end{gathered}
$$

\section{CONCLUSIONS}

A procedure to test whether compounds exhibit amountdependence, combined with the localization of the source of that effect within a GC-C-IRMS system, was established. Our experiments showed that, despite parameter optimization of crucial components such as the split/splitless injector, the amount-dependence of the $\delta^{13} \mathrm{C}$ values for the investigated phenols could not be eliminated completely. Nevertheless, our first stage optimization of the instrument parameters achieved a reduction of the amount-dependence from about 4 to $2 \%$ in the range of 4 to $80 \mathrm{ng}$ injected. The correction of the $\delta^{13} \mathrm{C}$ values measured with optimized parameters is simpler and more precise, since linear or logarithmic functions describing the amount-dependent fractionation can be applied instead of the more complex functions that had to be used prior to optimization (Fig. 1).

In addition to thorough adjustment of each individual component of a GC-C-IRMS system, we strongly recommend to run a set of standards with increasing analyte amounts and known isotopic composition together with each batch. As the amount-dependence occurs at a matrix-sensitive point of the system, i.e., the split/splitless injector, care has to be taken when comparing standards with samples or different sample 
matrix types. In terms of the ultimate goal of any instrumental setup of a CSIA method, the elimination of amountdependent isotopic fractionation, mathematical corrections should only serve as provisional solution; however, they are very helpful for routine analysis. Although isotopic fractionation during split/splitless injection is a phenomenon already described in the literature ${ }^{5,11}$ publications rarely give the exact injection conditions, i.e. liner type, needle, injection protocol, splitless time. Citing the exact parameters would be of tremendous help for others trying to apply the method. Furthermore, stating whether the method was tested for amount-dependent isotopic fractionation and whether or not the data are corrected for this effect would be of additional importance to assess the analytical quality and give broad awareness of this phenomenon.

\section{Acknowledgements}

The authors wish to thank T. Gonter and K. Jeschke for help in the lab and A. Wetzel for technical assistance. Funding for this research was provided the German Research Foundation (Ze 154/46-1) sponsoring the stable isotope facility in Bayreuth. K. Kaiser is thanked for fruitful discussions. The detailed and instructive comments of an anonymous reviewer made it possible to substantially improve the earlier version of this manuscript.

\section{REFERENCES}

1. Lichtfouse E. Rapid Commun. Mass Spectrom. 2000; 14: 1337.

2. Meier-Augenstein W. J. Chromatogr. A 1999; 842: 351.

3. Meier-Augenstein W. LC/GC Interntl. 1997; 10: 17.

4. Werner RA, Brand WA. Rapid Commun. Mass Spectrom. 2001; 15: 501.

5. Glaser B, Amelung W. Rapid Commun. Mass Spectrom. 2002; 16: 891.

6. Merritt DA, Freeman KH, Ricci MP, Studley SA, Hayes JM. Anal. Chem. 1995; 67: 2461.

7. Ricci MP, Merritt DA, Freeman KH, Hayes JM. Org. Geochem. 1994; 21: 561.

8. Brenna JT, Corso TN, Tobias HT, Caimi RJ. Mass Spec. Rev. 1997; 16: 227.

9. Rieley G. Analyst 1994; 119: 915.

10. Hall JA, Barth JAC, Kalin RM. Rapid Commun. Mass Spectrom. 1999; 13: 1231.

11. Meier-Augenstein W, Watt PW, Langhans C-D. J. Chromatogr. A 1996; 752: 233.

12. Merritt DA, Hayes JM. Anal. Chem. 1994; 66: 2336.

13. Ohlsson KEA, Wallmark PH. Analyst 1999; 124: 571.

14. Schweizer M, Fear J, Cadisch G. Rapid Commun. Mass Spectrom. 1999; 13: 1284.

15. Grob K (ed). Split and Splitless Injection for Quantitative Gas Chromatography: Concepts, Processes, Practical Guidelines, Sources of Error (4th edn). Wiley-VCH: Weinheim, 1999.

16. Habfast K. Advanced Isotope Ratio Mass Spectrometry I: Magnetic Isotope Ratio Mass Spectrometers. In Modern Isotope Ratio Mass Spectrometry, Platzner IT (ed). Wiley \& Sons Ltd: 1997; 11.

17. Meier-Augenstein W. Rapid Commun. Mass Spectrom. 1997; 11: 1775.

18. Goñi MA, Eglinton TI. Org. Geochem. 1996; 24: 601. 